\title{
Design and Implementation on Physics Experiment Equipment Management System
}

\author{
Xiang TENG \\ Basic Teaching and Research Institute, Bohai University, Jinzhou, China
}

\begin{abstract}
The rapid development of computer technology for university physics laboratory management has brought new techniques, management work of laboratory informatization, digital, networked, intelligent level gradually increased. In this paper, the use of computer technology to design the college physics experiment equipment management system, improve the efficiency and quality of equipment management. First, the system uses the SSH architecture, and is represented by the figure; then, database design, elaborated the system design principle of database, design the data table that is the most important of the system equipment information data table; Finally, module design, mainly include: equipment management module, equipment enter warehouse and out of library modules, equipment maintenance module, and so on. To achieve equipment entry, add, modify, query, statistics, delete, and maintenance management.
\end{abstract}

KEYWORD: Experiment equipment; SSH framework; Database; Function

\section{INTRODUCTION}

With the continuous development of productive forces, the progress of science and technology, the continuous improvement of network technology, the increasing demand for information, the computer has become indispensable tools in our daily life. The laboratory is the indispensable basic unit of all schools, research institutions. The equipment management is the school laboratory management in an extremely important content. With the expansion of the university the rapid development of laboratory construction, a large number of experiments instrument and equipment continue to purchase, school laboratories and asset management department over the years, handmade style management approach not only labor-intensive, low work efficiency, but also may make the equipment management confusion. Manual processing methods have been unable to meet the needs of modern management, the urgent need to achieve the network management of equipment [1].

In the physics lab, laboratory equipment is the guarantee of completing the experiment, laboratory equipment management is a tedious job. For example, Such as a variety of resistive and capacitive components of circuit experiment, chips, due to the characteristics of small size, easy to be lost and difficult to find, often to normal experimental bring some trouble, resulting in low work efficiency. In addition, some of the instruments or equipment due to loan or damage, did not timely record lead to lose, but also caused the loss of the laboratory [2]. Therefore, using computer management as the means, to develop a set of equipment management system, has the necessity and the practical significance. In this paper, in order to better achieve the scientific management of equipment facilities, design experiment equipment management system. The system realizes the rationalization, standardized, systematic of equipment management, to improve work efficiency of management, to meet the requirements of physics experiment. And because the use of multilayer database technology, the system has a certain stability, scalability and maintainability.

\section{TECHNICAL ARCHITECTURE}

Currently SSH architecture is the most widely used Java EE enterprise applications integrated development model, SSH architecture integrates Struts, Spring and Hibernate three popular open source framework, each of these three frame has its own characteristics, were respectively applied to the system for the presentation layer, business logic and data persistence layer, through the interface to communicate between layers. Using this integrated framework model developed software management 
systems to reduce the coupling of system code, with high scalability, high performance and high security features, improve maintainability of the system, so that Java EE development faster and more efficient.

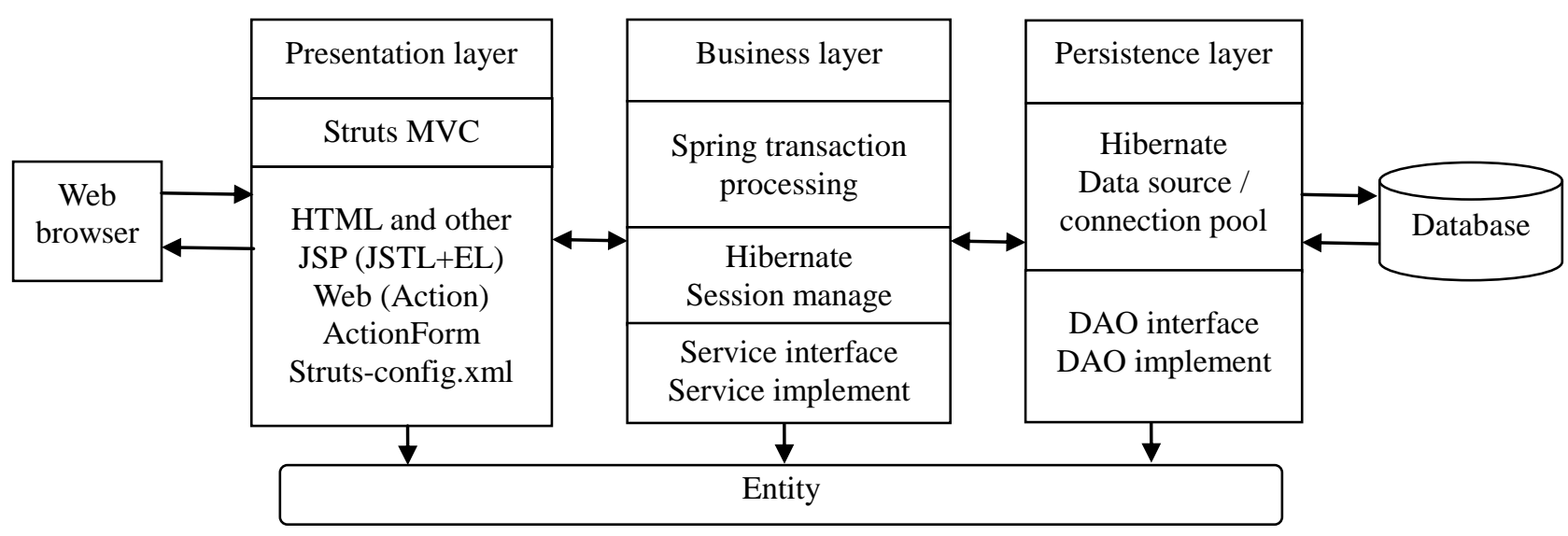

Fig. 1. SSH framework architecture

SSH framework architecture shown in Fig. 1. Struts are responsible for MVC separation and implementing web presentation layer, for process control. ActionFormBean receive that webpage form submitted data, and then processed through Action, and then Forward to the corresponding page. Action is defined in the struts-config.xml, loaded by ActionServlet. Spring is responsible for managing the business layer, business logic processing and circulation, spring with DAO (Data Access Objects) way to interact with data sources. Business Service Classes for Action provides a unified interface calls, encapsulate data access object DAO of persistence layer. Hibernate responsible for data persistence, encapsulates the basic operations of underlying for background database [3].

System's basic business processes are [4]: in the presentation layer, first through the JSP page implement interactive interface, charge transfer request and receive a response, and the Struts according to the configuration file (strutsconfig.xml) will ActionServlet received Request delegated to the appropriate Action processing. In the business layer, mainly dependent on the Spring Framework provides container for the completion of Strut, and Hibernate "seamless" connection. Spring IoC container of management services component is responsible for providing business model component and that component collaboration object data processing (DAO) component for Action to complete the business logic, and provides transaction processing, buffer pools and other containers components to improve system performance and ensure data integrity. And in the persistence layer, mainly related to the operation of the database, complete the operation of the database, add, delete, modify, search, update and so on.
Depends on the Hibernate object mapping and database interaction, handling DAO components requested data, and returns the processing results.

\section{DATABASE DESIGN}

Database design is critical data base, is also an important part of rational distribution of the whole system. It directly affects the efficiency of the system, stability and maintainability. The core of equipment management system is processed and stored data, so the core work of equipment management system analysis and design phase is to design the structure and implementation of the database, to determine the system needs to use the database structure. It provides data base for equipment management system, forming a data warehouse. But also which lays the foundation for systems business classification and delineation [5]. In order to shorten the development cycle and improve system performance, must carry on the database design, database design principles of the system are: data objects clearly mutual relations, consistent with the business logic functions; database system designed to facilitate the transfer, minimize code rewriting; storage structure reasonable, reduce duplication; named to "see the name know integrity"; maintain the integrity of data definitions; ensure the security of the database contents; definition of data table to meet the national standard or industry standards; choose good design patterns to optimize the database structure, easy to maintain; scale of database data records to be moderate, not too big, to improve the efficiency of data processing. Experimental equipment information table is shown in Table 1. 
Table 1. Experiment equipment information table

\begin{tabular}{|c|c|c|c|c|c|c|c|}
\hline Fields NO & Fields Name & Fields Type & Fields Width & Fields NO & Fields Name & Fields Type & Fields Width \\
\hline 1 & EquipID & Varchar & 20 & 10 & IntDate & Datetime & 8 \\
\hline 2 & EquipName & Varchar & 20 & 11 & OutDate & Datetime & 8 \\
\hline 3 & EquipType & Varchar & 20 & 12 & StockNumber & Varchar & 20 \\
\hline 4 & Specifications Model & Varchar & 20 & 13 & Balance Amount & Varchar & 20 \\
\hline 5 & SupplierID & Varchar & 20 & 14 & OutNumber & Nvarchar & 20 \\
\hline 6 & LaboratoryID & Varchar & 20 & 15 & IntNumber & Nvarchar & 20 \\
\hline 7 & EquipPrice & Momey & 4 & 16 & UseStatus & Nvarchar & 50 \\
\hline 8 & OutID & Varchar & 20 & 17 & CheckDate & Datetime & 8 \\
\hline 9 & IntID & Varchar & 20 & 18 & Remark & Varchar & 50 \\
\hline
\end{tabular}

The system uses SQL Server 2008; provides a comprehensive and scalable data warehouse platform, which can use a separate analysis and store carried powerful analysis, in order to meet thousands of users in a few megabytes of data needs. Trusted, can be very high security, reliability and scalability to run their most mission-critical applications; efficient, can reduce the time and cost to develop and manage their data infrastructure; intelligent, providing a comprehensive platform that can send him to observe and information at the time of users need [6].

\section{FUNCTION MODULE DESIGN}

The system mainly include: user management module, equipment procurement module, equipment management module, enter and out of warehouse modules, maintenance module, reservation module, equipment scrapped module. System function modules are shown in Fig. 2. The following describes the equipment management module:

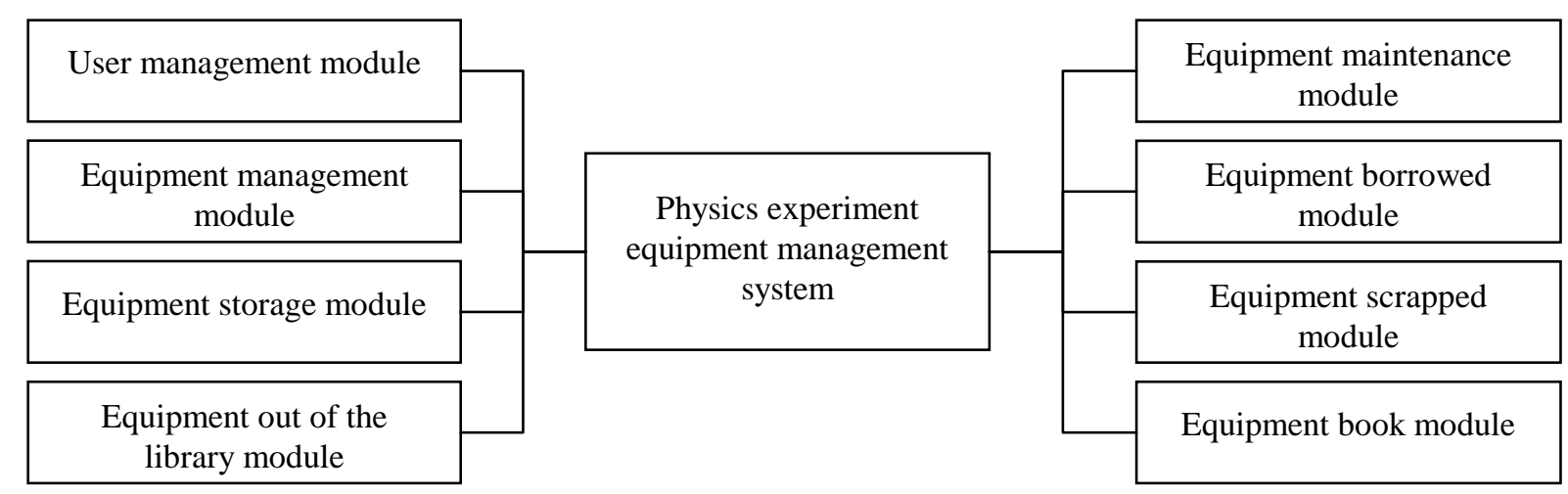

Fig. 2. Function module of equipment management system

Experiment equipment management module. This module belongs to all types of laboratory to classify register to enable the students and teacher query experimental equipment conditions. Mainly to achieve equipment add, modify, query, statistics, delete and other data management. This part by administrator according to the attribute information of equipment number, classification, equipment and related information on entry and assign it to a specific laboratory management. When buying new equipment and equipment scrapped, to add and delete, equipment lending and return, enter and out of warehouse to modify [7]. Teachers can view equipment information, but cannot do modify. Depending on the status of courses required equipment, for equipment were scheduled, if the number of equipment is insufficient, then submit purchase requisitions through the system.

Equipment maintenance module, equipment outdated, system based on the equipment production time and shelf life tracking, once expired, then automatically generates the expired warning, the administrator according to the prompt, its treatment, after verifying accurate, its properties are automatically become scrap, and re-modify warehouses number [8]. Users for fault equipment apply to repair, administrator designated repair personnel for maintenance. System recording repaired conditions, including repaired equipment types, fault phenomena, and so on, for future reference. 


\section{CONCLUSION}

Physics experiment equipment management system is to use a computer in the lab facilities, equipment, carry information and network management, it is necessary to combine the modern information technology and experiment equipment management. Due to the heavy workload of the previous, so easy to go wrong, scribbled unclear, modify inconvenient and difficult query, hard statistics, inconvenient to carry, when saving easy to damage these drawbacks. Now it can completely get rid of the traditional accounting management style. In the management process, particularly in procurement management, storage and out of warehouse management, experiment equipment reservation, equipment query and data statistics, and others, have brought great convenience, greatly improving the efficiency of the equipment administrator. This system can be applied not only to the experimental equipment management, can also be applied to other aspects of university equipment management, such as audiovisual equipment, PE equipment, musical instruments, art equipment management.

\section{ACKNOWLEDGEMENT}

This work is supported by "Teaching Reform Project of Bohai University: Research and practice on modern physics virtual experiment simulation system of development.

\section{REFERENCES}

[1] Y. X. Song, "Design and Implementation of College Open Laboratory Management Platform," Tianjin Normal University, 2008.

[2] Z. B. Chen, W. Chen, "Design and Implementation of Laboratory Equipment Management System," Journal of Fuzhou University, vol. 36, no. supp, pp. 180-183, 2008.

[3] J. G. Guo, "Work and Grade Management System Based on SSH," Microelectronics and Computer, vol. 27, no. 8, pp. 156-160, 2010.

[4] Baidu, "SSH Framework," http://baike.haosou.com/doc/5443762.html, 2015-1-11.

[5] B. X. Wang, "Design and Implementation of the Shengli Oilfield Down hole Equipment Management System," Tianjin University, 2012.

[6] Baidu, "SQL Server 2008," http://baike.baidu.com/link?url=4cXP7X4x3CWKF27wrkr bxXKxckwrgh-hjjhtZ9NiMehSvy4Puwcqffq6EXtuFMRjrQJQMvrkZzi92Um6 Pse6_, 2015-1-11.

[7] X. J. Shen, "The Design and Implementation of Open Laboratory Management System in Higher Vocational College," University of Electronic Science and technology, 2013.

[8] R. Hu, "Research and Development of the Communication Equipment Management System Based on C/S and B/S," Chongqing University, 2005. 